\title{
Remission, low disease activity and improvement of pain and function in psoriatic arthritis patients treated with IL-12/23 and IL-17 inhibitors. A multicenter prospective study
}

\author{
F.M. Perrotta' 1 A. Delle Sedie' ${ }^{2}$, S. Scriffignano', P. Volpe ${ }^{3}$, E. Cordisco ${ }^{4}$, \\ N. Milano ${ }^{4}$, M. Gabini ${ }^{3}$, E. Lubrano ${ }^{1}$ \\ 'Academic Rheumatology Unit, Dipartimento di Medicina e Scienze della Salute "Vincenzo Tiberio", \\ Università degli Studi del Molise, Campobasso; 2 U.O. Reumatologia, Azienda Ospedaliera-Universitaria \\ Pisana. Pisa; "UOC Reumatologia, Presidio Ospedaliero "Santo Spirito". Pescara; \\ "UOC di Medicina, Ambulatorio di Reumatologia, Ospedale "San Timoteo", Termoli, Italy
}

\section{SUMMARY}

The development of new biologic and targeted synthetic DMARDs can lead to good disease control. The aim of the present study was to assess the rate of remission and low disease activity, and the improvement of pain and function, in psoriatic arthritis (PsA) patients treated with new anti-IL-12/23 and anti-IL-17 biologic agents.

A prospective 6-month study was performed. Patients fulfilling the CASPAR criteria for PsA that started ustekinumab, secukinumab and ixekizumab were enrolled and prospectively followed in a setting of clinical practice. Patients were considered in minimal disease activity (MDA), when they met at least 5/7 of the criteria previously defined. DAPSA score $\leq 4$ was also evaluated as a remission criterion. Pain on VAS, PtGA and HAQ were also assessed in all patients. Patients achieving MDA were compared to non-MDA to identify outcome predictive factors.

Of the 70 patients treated with ustekinumab, secukinumab and ixekizumab, at baseline, no patients were in MDA or had a DAPSA score $\leq 4$. Ten patients $(14.2 \%)$ were lost during the follow-up. After 6 months, MDA was achieved in $22(31.4 \%)$ patients. DAPSA $\leq 4$ was achieved in $17(24.2 \%)$ patients. Significant improvement in pain, PtGA and HAQ was also found. Patients naïve to anti-TNF treatment achieved more frequently MDA compared to anti-TNF-experienced patients. Male sex, high levels of CRP and absence of comorbidities were found to be predictors of MDA.

In our prospective observational study, MDA was achieved in $31.4 \%$ and DAPSA remission in $24.2 \%$ of patients treated with inhibitors of IL-12/23 and IL-17, thus making this target achievable in PsA patients treated with these drugs.

Key words: Psoriatic arthritis; remission; anti-IL12/23; anti-IL-17.

Reumatismo, 2020; 72 (1): 52-59

Corresponding author: Ennio Lubrano

Dipartimento di Medicina e di Scienze per la Salute "Vincenzo Tiberio", Università del Molise, Via Giovanni Paolo II,

C/da Tappino, 86100 Campobasso, Italy. E-mail: enniolubrano@hotmail.com

\section{INTRODUCTION}

soriatic arthritis (PsA) is a chronic inflammatory disease associated with psoriasis with a prevalence rate of 133 every 100,000 subjects and an incidence, in Italy, of about 22/100,000 per year (1-3). The peripheral joint involvement of PsA is progressive in the majority of patients, with the presence of inflammatory and structural changes even at entheseal and axial sites. Moreover, patients with PsA can have functional impairment in daily activities, depression, fatigue and reduced quality of life. Therefore, there is the need for optimal management and treatment strategies (4-6). In the context of this complex and multifaceted disease, remission or low disease activity are considered the treatment target leading, in general, to a better clinical and radiographic outcome (7). In 2010, Coates et al. developed a composite outcome measure (minimal disease activity - MDA) for patients with PsA that encompasses 
most of the disease domains and could be used to define a state of low disease activity (8). Furthermore, other disease activity measures and definitions of remission were developed, such as the disease activity index for psoriatic arthritis (DAPSA), which, given its ease of use, is often adopted in the clinical practice (9). Treatment with tumor necrosis factor $\alpha$ (TNF) blockers radically changed the management of PsA, showing efficacy in clinical trials and effectiveness in real life. In this context, the anti-TNF treatment proved to reduce disease activity parameters and radiographic progression, to be efficacious in the treatment of skin lesions and enthesitis and in the improvement of quality of life, leading, in the real life setting, to MDA or remission/low disease activity in $40-60 \%$ of patients (10-13). However, unmet needs still remain for patients resistant or intolerant to the anti-TNF treatment. With the development of new biologic drugs and small molecules that showed their efficacy in clinical trials (14-16), the possibility to induce a status of remission or low disease activity and to improve quality of life will be enhanced. In this scenario the aim of this prospective study was to investigate the possibility to achieve MDA or DAPSA remission in PsA patients treated with new biologics (inhibitors of IL-12/23 and IL-17) drugs in a real-life setting. Its secondary aim was to evaluate the improvement of pain and function and to identify predictors for the achievement of MDA.

\section{PATIENTS AND METHODS}

Patients were enrolled in four Rheumatology Units in Italy (Campobasso, Pescara, Termoli and Pisa). All patients fulfilled the ClASsification criteria for Psoriatic ARthritis (CASPAR) (16). Both patients naïve to TNF inhibitors or intolerant/resistant to TNF inhibitors were enrolled. All biologics were prescribed according to the recommendation of the Italian Society of Rheumatology (17). A written patient consent was obtained according to the declaration of Helsinki, and the study was approved by local ethical-scientific committee of the University of Molise (n. 0001-09-2017).

\section{PATIENT SELECTION}

All of the patients with PsA started on ustekinumab (UST) (an initial dose of 45 mg administered subcutaneously, followed by a $45 \mathrm{mg}$ dose 4 weeks later, and then every 12 weeks thereafter for patients with body weight $\leq 100 \mathrm{Kg}$, or a dose of $90 \mathrm{mg}$ with the same regime for patients with body weight $>100 \mathrm{~kg}$ ), secukinumab (SEC), (300 $\mathrm{mg}$ by subcutaneous injection for patients with concomitant moderate to severe plaque psoriasis or who are anti$\mathrm{TNF} \alpha$ inadequate responders with initial dosing at Weeks $0,1,2,3$ and 4, followed by monthly maintenance dosing or $150 \mathrm{mg}$ for other patients) or ixekizumab (IXE) (160 $\mathrm{mg}$ by subcutaneous injection at Week 0 , followed by $80 \mathrm{mg}$ every 4 weeks thereafter) were considered potentially eligible for the study.

Exclusion criteria were:

1) age $<18$ years;

2) MDA status before the initiation of the biologic therapy or at the baseline visit. The enrolled patients were evaluated at baseline before starting the biologic treatment, and after 6 months of biologic treatment according to clinical practice. The choice of a specific agent was based on the clinical characteristic of PsA patients and on the patient and physician's preference.

\section{DATA COLLECTION}

Patient data collection included a detailed history, physical examination, current use of medications, presence of comorbidities (hypertension, type 2 diabetes, metabolic syndrome, cardiovascular diseases) and laboratory assessment. Age, gender, disease duration, smoking status and pattern of articular manifestations were also collected. The definition of axial disease was based on the presence of clinical (spinal inflammatory pain according to the Calin criteria) and/or radiological axial involvement (18). The clinical assessment included a number of tender joints (of the 68 assessed joints) and swollen joints (total of 66 joints), enthesitis and dactylitis. Enthesitis was measured using the Leeds Enthesitis In- 
dex (LEI), and dactilytis as present/absent or previous (19). All patients underwent a clinical examination of the skin by trained investigators that included the psoriasis area severity index (PASI) score and the body surface area (BSA) (20). Patients also completed self-reported questionnaires

Table I - Clinical characteristics of PsA patients $(n=70)$ treated with UST, SEC and IXE at baseline.

\begin{tabular}{|c|c|}
\hline Male / Female & $39 / 31$ \\
\hline Age; years (mean/SD) & $45.7(11.5)$ \\
\hline $\mathrm{BMI}$ & $27.5(24.7-33)$ \\
\hline Disease duration; years (median/IQR range) & $7 / 3.25-10$ \\
\hline $\begin{array}{l}\text { Articular manifestations (\%) } \\
\text { Axial } \\
\text { Peripheral arthritis } \\
\text { Enthesitis } \\
\text { Dactylitis }\end{array}$ & $\begin{array}{l}24.3 \\
95.7 \\
35.7 \\
21.4\end{array}$ \\
\hline $\begin{array}{l}\text { Extra-articular manifestations (\%) } \\
\text { Uveitis } \\
\text { IBD }\end{array}$ & $\begin{array}{c}1.4 \\
0\end{array}$ \\
\hline DAPSA (median/IQR range) & $21.1 / 13-29.7$ \\
\hline Tender Joint (median/IQR range) & $4 / 2-8$ \\
\hline Swollen Joint (median/IQR range) & $1 / 0-2$ \\
\hline ESR; mm/1st hr (median/IQR range) & $21.5 / 10.7-34$ \\
\hline CRP; mg/L (median/IQR range) & $3.6 / 2-8.8$ \\
\hline PtGA (0-100) (median/IQR range) & $70 / 50-80$ \\
\hline VAS pain (0-100) (median/IQR range) & $70 / 40-80$ \\
\hline VAS Physician (0-100) (median/IQR range) & $60 / 50-70$ \\
\hline $\mathrm{HAQ}$ (median/IQR range) & $1 / 0.3-1.2$ \\
\hline PASI (median/IQR range) & $3 / 1-8.4$ \\
\hline $\mathrm{BSA} \%$ (median/IQR range) & $4(1-7)$ \\
\hline LEI (median/IQR range) & $1 / 0-1$ \\
\hline \multicolumn{2}{|l|}{ Previous treatment } \\
\hline Previous anti-TNF treatment, $\mathrm{n}(\%)$ & $35(50)$ \\
\hline Previous Ustekinumab treatment, $\mathrm{n}(\%)$ & $6(8.5)$ \\
\hline Previous Apremilast treatment, $\mathrm{n}(\%)$ & $1(1.4)$ \\
\hline $\begin{array}{l}\text { Concomitant csDMARDs, n (\%) } \\
\text { - Methotrexate } \\
\text { - Sulfasalazine } \\
\text { - Leflunomide }\end{array}$ & $\begin{array}{l}30(42.8) \\
26(86.6) \\
3(10) \\
1(3.4)\end{array}$ \\
\hline Concomitant systemic steroids & $5(7.1)$ \\
\hline
\end{tabular}

SD: standard deviation; BMI: Body mass index; IQR: interquartile range; IBD: inflammatory bowel diseases; DAPSA: Disease activity score for psoriatic arthritis; ESR: erythrosedimentation rate; CRP: C reactive protein; PtGA: patient's global assessment; VAS: visual analogue scale; HAQ: health assessment questionnaire; PASI: psoriasis area severity index; BSA: body surface area; LEl: Leeds enthesitis index; TNF: tumor necrosis factor. csDMARDs: conventional synthetic Disease Modifying Anti-Rheumatic Drugs. including the health assessment questionnaire (HAQ) (21), and a global (PtGA) and pain assessment on Visual Analogic Scale (VAS 0-100 mm) (22). Physician's global evaluation of disease on VAS was also recorded. Laboratory investigations included $\mathrm{C}$ reactive protein (CRP). Clinical data were collected at baseline and at 6 months follow-up.

\section{RESPONSE CRITERIA}

MDA was identified according to Coates et al. (8) and assessed at baseline and after 6 months of treatment. MDA was deemed as achieved, when patients met $5 / 7$ of the following criteria: tender joint count $\leq 1$; swollen joint count $\leq 1$; PASI $\leq 1$ or $\mathrm{BSA} \leq 3$; patient pain visual analogue scale (VAS) score of $\leq 15$; PtGA on VAS score of $\leq 20$; HAQ score $\leq 0.5$; and tender entheseal points $\leq 1$. DAPSA score was identified according to Schoels et al. (9). DAPSA was calculated by adding up the number of tender and swollen joints, VAS pain, PtGA and CRP (mg/dl). A DAPSA score $\leq 4$ was defined remission, while a DAPSA score $\leq 14$ defined low disease activity. DAPSA score between 14 and 28 identified moderate disease activity, while DAPSA $>28$ high disease activity. The rate of patients achieving a pain $\mathrm{VAS} \leq 15, \mathrm{PtGA} \leq 20$ and $\mathrm{HAQ} \leq 0.5$ was also evaluated.

\section{STATISTICAL ANALYSIS}

The results were expressed as median $\left(25^{\text {th }}-75^{\text {th }}\right.$ percentile $)$. A percentage was used to assess the rate of patients achieving MDA or DAPSA remission at baseline and after 6 months. Non-responder imputation was used to evaluate the rate of patients achieving MDA or DAPSA remission assuming treatment failure for all dropouts (missing patients). Categorical variables were analyzed by $\chi$-square test with Yates' correction or Fisher's exact test. The significance of the differences was determined using the MannWhitney test for unpaired samples and Wilcoxon's test for paired samples. The probability of achieving MDA was ex- 


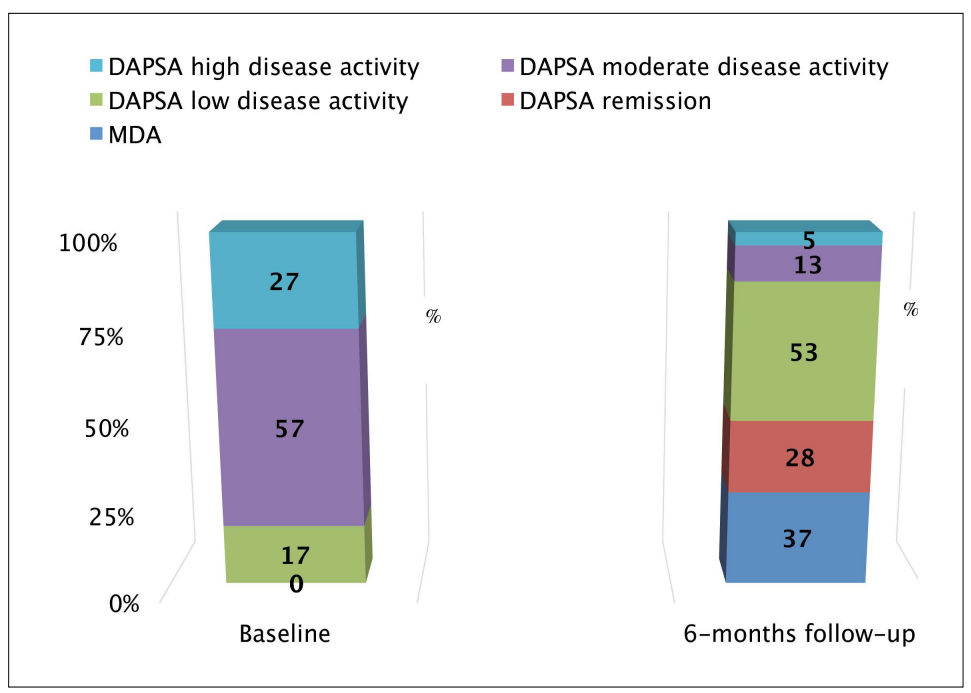

Figure 1 - Percentage of patients in MDA, DAPSA remission, DAPSA low disease activity, DAPSA moderate disease activity and DAPSA high disease activity at baseline and after 6 months of UST, SEC and IXE treatment $(p<0.05$ for each comparison with baseline values).

plored using the odd ratio (OR - lower and upper $95 \% \mathrm{CI}$ ) of outcome relative to the main variables. For this purpose, male/female, the values of age $(\leq$ or $>50$ years), disease duration ( $\leq$ or $>2$ years), $\mathrm{BMI}(\leq$ or $>30)$, skin involvement (BSA $\leq 3$ vs $\mathrm{BSA}>3$ ), axial involvement (absence vs presence), CRP (low $\leq 0.5$, high $>0.5$ $\mathrm{mg} / \mathrm{dL}$ ), comorbidities (present vs absent) were categorized. $P$ values $<0.05$ were considered significant.

\section{RESULTS}

At baseline, 70 patients were enrolled and treated with UST $(n=20)$, SEC $(n=42)$ and IXE $(n=8)$. No patients had a DAPSA score $\leq 4$ at baseline, while $12(17.1 \%)$ had a DAPSA score $\leq 14$. Furthermore, 40
$(57.1 \%)$ had moderate disease activity and $18(26.8 \%)$ high disease activity according to DAPSA scores. Table I shows the demographic and clinical characteristics of the study population. Ten $(14.2 \%)$ patients were lost during the follow up (3 treated with UST, 6 treated with SEC, 1 treated with IXE). After 6 months, MDA was achieved in $22(36.6 \%)$ out of the remaining 60 patients. DAPSA $\leq 4$ was achieved in $17(28.3 \%)$ patients and DAPSA $\leq 14$ in 32 (53.3\%) (Figure 1). Using non-responder imputation MDA, DAPSA $\leq 4$ and DAPSA $\leq 14$ were achieved respectively in $31.4 \%$, $24.2 \%$ and $45.7 \%$ of patients. Patients naïve to anti-TNF treatment achieved more frequently MDA (47.3\%) compared to anti-TNF-experienced patients $(31.7 \%)$. No significant differences in the achievement

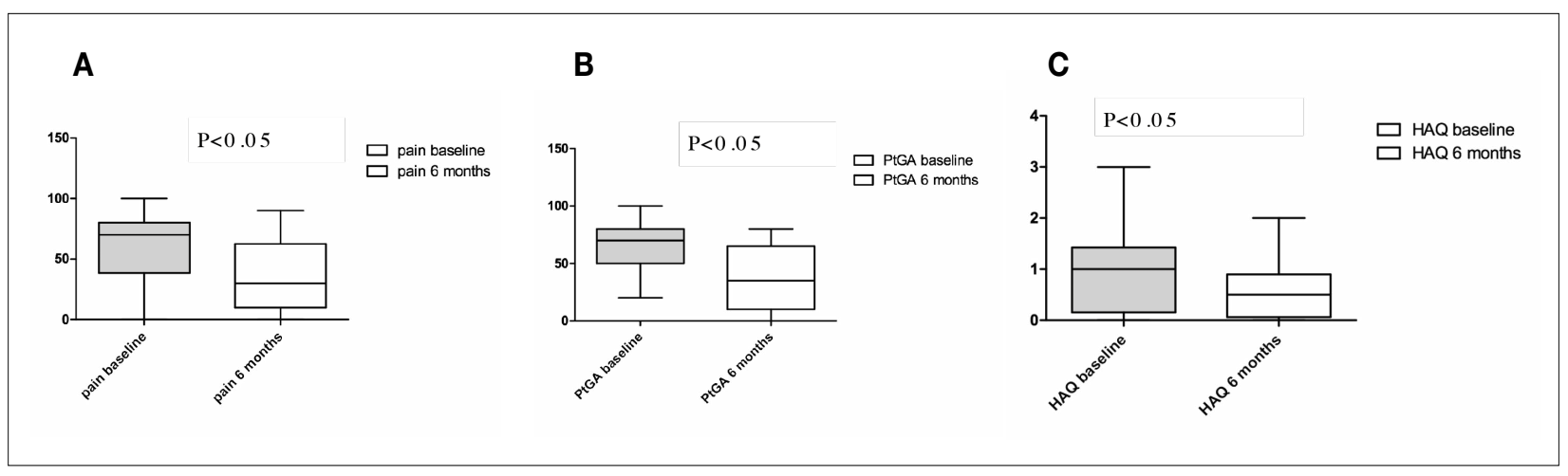

Figure 2 - Values (box and whiskers) of pain on VAS (A), PtGA (B) and HAQ (C) in patients with PsA at baseline and after 6 months of treatment with UST, SEC and IXE. 
Table II - ORs (95\% Cl) of achieving MDA at month 6 in patients treated with UST, SEC and IXE according to the baseline variable values.

\begin{tabular}{|l|c|c|}
\hline & OR (95\% Cl) & p value \\
\hline $\begin{array}{l}\text { Gender } \\
\text { Male vs female }\end{array}$ & $\begin{array}{c}11.2 \\
(2.5-48.7)\end{array}$ & $<0.01$ \\
\hline $\begin{array}{l}\text { Age: } \\
\leq 50 \text { yrs vs }>50 \text { yrs }\end{array}$ & $\begin{array}{c}0.94 \\
(0.29-2.9)\end{array}$ & 0.76 \\
\hline $\begin{array}{l}\text { Disease duration: } \\
\leq 2 \text { yrs vs }>2 \text { yrs }\end{array}$ & $\begin{array}{c}0.47 \\
(0.14-1.53)\end{array}$ & 0.21 \\
\hline $\begin{array}{l}\text { BMl } \\
\leq 30 \text { vs }>30\end{array}$ & $\begin{array}{c}0.48 \\
(0.13-1.75)\end{array}$ & 0.25 \\
\hline $\begin{array}{l}\text { Skin involvement } \\
\text { BSA } \leq 3 \text { vs BSA>3 }\end{array}$ & $\begin{array}{c}1.02 \\
(0.3-3.8)\end{array}$ & 0.76 \\
\hline $\begin{array}{l}\text { Axial involvement } \\
\text { absence vs presence }\end{array}$ & $\begin{array}{c}0.39 \\
(0.1-1.52)\end{array}$ & 0.13 \\
\hline $\begin{array}{l}\text { LEI } \\
0 \text { vs } \geq 1\end{array}$ & $\begin{array}{c}0.4 \\
(0.1-1.5)\end{array}$ & 0.17 \\
\hline $\begin{array}{l}\text { CRP: } \\
\text { high vs low }\end{array}$ & $\begin{array}{c}3.9 \\
(1.07-14)\end{array}$ & 0.03 \\
\hline $\begin{array}{l}\text { Comorbidities (all) } \\
\text { presence vs absence }\end{array}$ & $\begin{array}{c}0.20 \\
(0.05-0.72)\end{array}$ & 0.01 \\
\hline $\begin{array}{l}\text { Biologic monotherapy vs combination } \\
\text { with csDMARDs }\end{array}$ & $\begin{array}{c}0.89 \\
(0.23-2.4)\end{array}$ & 0.6 \\
\hline
\end{tabular}

ODs: odds ratio, UST: Ustekinumab; SEC: Secukinumab; IXE: Ixekizumab; MDA: minimal disease activity; BMI: body mass index; BSA: body surface area; LEl: Leeds enthesitis index; CRP: C reactive protein; csDMARDs: conventional synthetic disease modifying anti-rheumatic drugs.

of MDA or DAPSA cut-offs were found among patients treated with IL-12/23 inhibitor (MDA 30\%, DAPSA remission $20 \%$ ) and IL-17 inhibitors (MDA 40\%, DAPSA remission $26 \%$ ), but the study was underpowered to detect such a difference. Finally, 6 out of the 12 patients with baseline DAPSA LDA achieved a DAPSA remission after 6 months.

DAPSA, BSA, LEI, CRP and number of tender and swollen joints significantly decreased after 6 months. The improvement (median/IQR) of pain on VAS, PtGA and HAQ compared to baseline was -30 $(-10 /-37),-30(-10 /-45)$ and $-0.125(0 /-$ $0.5)$ respectively (Figure 2). Finally, $45 \%$, $41.6 \%$ and $35 \%$ of patients achieved a pain on $\mathrm{VAS} \leq 20, \mathrm{PtGA} \leq 20$ and a HAQ $\leq 0.5$. The association of MDA with clinical characteristics of patients was studied. Table II shows the Odds Ratio (95\% CI) of achieving MDA at 6 months according to the baseline characteristics. Male gender, high CRP levels and absence of comorbidi- ties were independent predictors of MDA. However, the results achieved are limited, because the tests used did not allow for any correction for confounders.

\section{DISCUSSION}

Remission or low disease activity status is the treatment goal in chronic inflammatory arthritis. In spondyloarthritis and in particular in PsA, a condition of remission or low disease activity using biologic anti-TNF drugs was an achievable target $(11,23,24)$. In our study, $36.6 \%$ of the 70 initial PsA patients treated with new anti-interleukins drugs were in MDA and $28.3 \%$ in DAPSA remission after 6 months of treatment. Furthermore, over $50 \%$ of patients achieved a status of DAPSA LDA. These results are in keeping with previous studies on anti-TNF $(25,26)$. Other studies in a setting of clinical practice reported a similar response rate. In our previous prospective studies in patients treated 
with anti-TNF, naïve to biologics, MDA and DAPSA remission were achieved respectively by $58.6 \%$ and $16 \%$ of patients after 8 months of treatment (11). However, in this study, the characteristics of patients enrolled (anti-TNF naïve) and the different criteria to assess DAPSA remission could explain these differences. With regards to new biologic treatment, only few studies assessed the achievement of MDA and remission in a setting of clinical practice and the available evidence mainly comes from clinical trials. In a recent study, Queiro et al. showed that $54 \%$ of patients treated with UST achieved MDA in a setting of clinical practice (27). A recent study on UST survival in PsA showed similar data with a rate of patients achieving DAPSA remission of $34 \%$ (28). Data for SEC and IXE are scarce. In clinical trials, using data coming from FUTURE 2 study with SEC, authors reported that, at 16 weeks, MDA and DAPSA remission were reached overall by $34 \%$ and $20 \%$ of anti-TNF naïve patients respectively (29).

In a recent prospective study on 20 PsA patients receiving SEC, $46 \%$ reached MDA and $52 \%$ DAPSA low disease activity (defined as DAPSA <14) (30).

For IXE, data coming from SPIRIT-2 trial showed that about $35 \%$ and $20 \%$ of patients treated with the IXE every four weeks achieved MDA and DAPSA remission respectively. Moreover, these drugs are efficacious regardless the use of background DMARDs (31). Recently, the SPIRIT-H2H trial compared head-to-head IXE and adalimumab monotherapy in PsA patients naïve to biologic drugs. In this study MDA and DAPSA remission were achieved by $47.7 \%$ and $26.5 \%$ of patients treated with IXE and by $35.5 \%$ and $18 \%$ of patients treated with adalimumab, respectively (32).

The results of our study are in line with those published in the literature, showing a good efficacy of the three drugs which inhibit an important pathogenetic pathway. Although no other head to head study was performed, our study confirmed that a good proportion of patients can achieve these important outcomes.
The finding that patients naïve to biologic treatment achieved significantly more frequently a condition of MDA and remission is supported by similar results in clinical trials (33). Improvement in pain and function is also of crucial importance for PsA patients. Previous reports coming from phase III trials on anti-IL-17 drugs described significant improvement of HAQ and pain $(33,34)$. In this study we found similar results, with a significant improvement in these important patient-reported outcomes.

Another interesting point is that, although $17 \%$ of patients had DAPSA LDA at baseline evaluation, we decided to introduce or change the biologic therapy for different reasons:

firstly, some patients had residual disease activity in some domains (e.g. skin or joints). In fact, 10/12 of patients with DAPSA LDA did not meet the MDA criteria. Furthermore, in some patients (young patients, extensive psoriasis despite a good control of joint symptoms) we aimed to achieve remission (DAPSA $<4$ ) by using a different treatment option. In fact, 6 out of the 12 patients with baseline DAPSA LDA achieved a DAPSA remission after 6 months.

In our study, male sex, high serum levels of CRP and absence of comorbidities were found to be associated with a better response to UST, SEC and IXE. This reflects the results of anti-TNF treatment in both PsA and axial Spondyloarthritis (11, 35, 36). Furthermore, the presence of comorbidities was associated with a lower probability of achieving MDA. Interestingly, obesity does not seem to be associated with a lower rate of MDA in our cohort, as showed in Table II.

Our study had some limits: firstly, the relative low number of patients treated with each drug does not allow to find any differences in the efficacy of each treatment. However, the aim was to evaluate the efficacy of non anti-TNF biologics as a class of molecules. Secondly, we evaluated the efficacy in the short term (6 months), therefore longer-term follow-up is need to confirm these results. 


\section{CONCLUSIONS}

Early DAPSA remission or MDA after 6 months is achievable in more than one third of patients, who are male and have high baseline CRP values and absence of comorbidities, which appear to be potential predictors of better response. More studies are needed to evaluate long-term remission and, in particular, if this state could be lost due to disease flares or maintained after reducing or stopping the biologic treatment.

\section{Disclaimer}

Authors declare that no parts of the manuscript have been reproduced from elsewhere.

\section{Conflict of interest}

The authors declare that there are no conflicts of interest.

\section{REFERENCES}

1. Chandran V, Raychaudhuri SP. Geoepidemiology and environmental factors of psoriasis andpsoriatic arthritis. J Autoimmun. 2010; 34: 314-21.

2. Scotti L, Franchi M, Marchesoni A, Corrao G. Prevalence and incidence of psoriatic arthritis: A systematic review and meta-analysis. Semin Arthritis Rheum. 2018; 48: 28-34.

3. De Socio A, Perrotta FM, Grasso GM, Lubrano E. Incidence of rheumatoid arthritis, psoriatic arthritis and polymyalgia rheumatica in an inland area of central Italy: results of the CAMPO-RHE study. Postgrad Med. 2018; 130: 137-41.

4. Perrotta FM, Astorri D, Zappia M, et al. An ultrasonographic study of enthesis in early psoriatic arthritis patients naive to traditional and biologic DMARDs treatment. Rheumatol Int. 2016; 36: 1579-83.

5. Helliwell P, Coates L, Chandran V, et al. Qualifying unmet needs and improving standards of care in psoriatic arthritis. Arthritis Care Res (Hoboken). 2014; 66: 1759-66.

6. Gladman DD. Disability and quality of life considerations. Psoriatic arthritis. In Gordon GB, Ruderman E, Editors. Psoriasis and psoriatic arthritis: an integral approach. Heidelberg: Springer- Verlag, 2005; 118-237.

7. Smolen JS, Braun J, Dougados M, et al. Treating spondyloarthritis, including ankylosing spondylitis and psoriatic arthritis, to target: recommendations of an international task force. Ann Rheum Dis. 2014; 73: 6-16.
8. Coates LC, Fransen J, Helliwel PS. Defining disease activity in psoriatic arthritis: a proposed objective target for treatment. Ann Rheum Dis. 2010; 69: 48-53.

9. Schoels MM, Aletaha D, Alasti F, Smolen JS. Disease activity in psoriatic arthritis (PsA): defining remission and treatment success using the DAPSA score. Ann Rheum Dis. 2016; 75: 811-8

10. Haddad A, Thavaneswaran A, Ruiz-Arruza I, et al. Minimal disease activity and anti-tumor necrosis factor therapy in psoriatic arthritis. Arthritis Care Res (Hoboken). 2015; 67: 8427.

11. Perrotta FM, Marchesoni A, Lubrano E. Minimal disease activity and remission in psoriatic arthritis patients treated with anti-TNF- $\alpha$ drugs. J Rheumatol. 2016; 43: 350-5.

12. Lubrano E, De Socio A, Perrotta FM. Comparison of Composite Indices Tailored for Psoriatic Arthritis Treated with csDMARD and bDMARD: A Cross-sectional Analysis of a Longitudinal Cohort. J Rheumatol. 2017; 44: 1159-64.

13. Lubrano E, Parsons WJ, Perrotta FM. Assessment of Response to Treatment, Remission, and Minimal Disease Activity in Axial Psoriatic Arthritis Treated with Tumor Necrosis Factor Inhibitors. J Rheumatol. 2016; 43: 918-23.

14. Lubrano E, Perrotta FM. Secukinumab for ankylosing spondylitis and psoriatic arthritis. Ther Clin Risk Manag 2016; 12: 1587-92.

15. Lubrano E, Perrotta FM. Beyond TNF Inhibitors: New Pathways and Emerging Treatments for Psoriatic Arthritis. Drugs 2016; 76 : 663-73.

16. Taylor W, Gladman D, Helliwell P, et al. CASPAR Study Group. Classification criteria for psoriatic arthritis: development of new criteria from a large international study. Arthritis Rheum. 2006; 54: 2665-73.

17. Marchesoni A, Olivieri I, Salvarani C, et al. Recommendations for the use of biologics and other novel drugs in the treatment of psoriatic arthritis: 2017 update from the Italian Society of Rheumatology. Clin Exp Rheumatol. 2017; 35: 991-1010.

18. Lubrano E, Spadaro A, Marchesoni A, et al. The effectiveness of a biologic agent on axial manifestations of psoriatic arthritis. A twelve months observational study in a group of patients treated with etanercept. Clin Exp Rheumatol. 2011; 29: 80-8.

19. Healy PJ, Helliwell PS. Measuring clinical enthesitis in psoriatic arthritis: assessment of existing measures and development of an instrument specific to psoriatic arthritis. Arthritis Rheum. 2008; 59: 686-91.

20. Fredriksson T, Pettersson U. Severe psoriasis-oral therapy with a new retinoid. Dermatologica 1978; 157: 238-44. 
21. Ranza R, Marchesoni A, Calori G, et al. The Italian version of the Functional Disability Index of the Health Assessment Questionnaire. A reliable instrument for multicenter studies on rheumatoid arthritis. Clin Exp Rheumatol. 1993; 11: 123-8.

22. Lubrano E, Perrotta FM, Parsons WJ, Marchesoni A Patient's Global Assessment as an Outcome Measure for Psoriatic Arthritis in Clinical Practice: A Surrogate for Measuring Low Disease Activity? J Rheumatol. 2015; 42: 2332-8.

23. Spadaro A, Lubrano E, Marchesoni A, et al. Remission in ankylosing spondylitis treated with anti-TNF- $\alpha$ drugs: a national multicentre study. Rheumatology (Oxford). 2013; 52: 1914-9.

24. Lubrano E, Perrotta FM, Marchesoni A, et al. Remission in non-radiographic axial spondyloarthritis treated with anti-tumor necrosis factor- $\alpha$ drugs: an Italian multicenter study. J Rheumatol. 2015; 42: 258-63.

25. Mease PJ, Heckaman M, Kary S, Kupper H. Application and modifications of minimal disease activity measures for patients with psoriatic arthritis treated with adalimumab: subanalyses of ADEPT. J Rheumatol. 2013; 40: 647-52.

26. Coates L, Helliwell P. Validation of Minimal Disease Activity Criteria for Psoriatic Arthritis Using Interventional Trial Data. Arthrit Care Res. 2010; 62: 965-9.

27. Queiro R, Brandy A, Rosado MC, et al. Minimal Disease Activity and Patient-Acceptable Symptom State In Psoriatic Arthritis: A RealWorld Evidence Study With Ustekinumab. J Clin Rheumatol. 2018; 24: 381-4.

28. Iannone F, Santo L, Bucci R, et al. Drug survival and effectiveness of ustekinumab in patients with psoriatic arthritis. Real-life data from the biologic Apulian registry (BIOPURE). Clin Rheumatol. 2018; 37: 667-75.

29. Coates LC, Nash P, Kvien T, et al. Secukinumab Provides Sustained Minimal Disease Activity (MDA) and Remission Related to Disease Activity Index for Psoriatic Arthritis (DAPSA): 2-Year Results from a Phase 3 Study (abstract 622). Arthritis Rheumatol. 2017; 69 (suppl 10).
30. Kampylafka E, d'Oliveira I, Linz C, et al. Resolution of synovitis and arrest of catabolic and anabolic bone changes in patients with psoriatic arthritis by IL-17A blockade with secukinumab: results from the prospective PSARTROS study. Arthritis Res Ther. 2018; 20: 153 .

31. Genovese MC, Combe B, Kremer JM, et al. Safety and efficacy of ixekizumab in patients with PsA and previous inadequate response to TNF inhibitors: week 52 results from SPIRIT-P2. Rheumatology (Oxford). 2018; 57: 2001-11.

32. Mease PJ, Smolen JS, Behrens F, et al. SPIRIT $\mathrm{H} 2 \mathrm{H}$ study group. A head-to-head comparison of the efficacy and safety of ixekizumab and adalimumab in biological-naïve patients with active psoriatic arthritis: 24-week results of a randomised, open-label, blinded-assessor trial. Ann Rheum Dis. 2019 [Epub ahead of print].

33. Mease PJ, Kavanaugh A, Reimold A, et al. Secukinumab in the treatment of psoriatic arthritis: efficacy and safety results through 3 years from the year 1 extension of the randomised phase III FUTURE 1 trial. RMD Open 2018. [Epub ahead of print].

34. Nash P, Kirkham B, Okada M, et al. SPIRITP2 Study Group. Ixekizumab for the treatment of patients with active psoriatic arthritis and an inadequate response to tumour necrosis factor inhibitors: results from the 24-week randomised, double-blind, placebo-controlled period of the SPIRIT-P2 phase 3 trial. Lancet 2017; 389: 2317-27.

35. Theander E, Husmark T, Alenius GM, et al. Early psoriatic arthritis: short symptom duration, male gender and preserved physical functioning at presentation predict favourable outcome at 5-year follow-up. Results from the Swedish Early Psoriatic Arthritis Register (SwePsA). Ann Rheum Dis. 2014; 73: 407-13.

36. Lubrano E, Perrotta FM, Manara M, et al.The Sex Influence on Response to Tumor Necrosis Factor- $\alpha$ Inhibitors and Remission in Axial Spondyloarthritis. J Rheumatol. 2018; 45: 195-201. 